\title{
Incidence and short-term outcome of acute lung injury in mechanically ventilated children
}

\author{
P. Dahlem*, W.M.C. van Aalderen*, M.E. Hamaker*, M.G.W. Dijkgraaf*, A.P. Bos*
}

Incidence and short-term outcome of acute lung injury in mechanically ventilated children. P. Dahlem, W.M.C. van Aalderen, M.E. Hamaker, M.G.W. Dijkgraaf, A.P. Bos. (C) ERS Journals Ltd 2003.

ABSTRACT: The aim of this study was to determine the incidence and short-term outcome of mechanically ventilated children suffering from acute lung injury (ALI) on a paediatric intensive care unit (PICU).

Between January 11998 and January 1 2000, all mechanically ventilated children were evaluated using the criteria of an American-European Consensus Conference.

Of the 443 children eligible for analysis, $44(9.9 \%)$ were diagnosed as suffering from ALI. Of these, $79.5 \%$ developed the acute respiratory distress syndrome (ARDS); $54.5 \%$ (24 of 44) fulfilled the ARDS criteria at inclusion and 25\% (11 of 44) later. PICU mortality for ALI was $27.3 \%$ (12 of 44) and within the ARDS subgroup $31.4 \%$ (11 of 35). Of the 12 children who died, 11 had ARDS; the main cause of death was cerebral damage (seven of 12).

Acute lung injury and acute respiratory distress syndrome are rare diseases on a paediatric intensive care unit with a high mortality. Most of the children with acute lung injury develop acute respiratory distress syndrome. In the acute respiratory distress syndrome subgroup, mortality is higher than in the acute lung injury nonacute respiratory distress syndrome subgroup. Further investigations should confirm prognostic factors (e.g. respiratory parameters) for prediction of outcome.

Eur Respir J 2003; 22: 980-985.
*Dept of Paediatrics, Emma Children's Hospital, and ${ }^{\#}$ Dept of Clinical Epidemiology and Biostatistics, Academic Medical Centre of the University of Amsterdam, Amsterdam, the Netherlands.

Correspondence: P. Dahlem

Paediatric Intensive Care Unit,

Academic Medical Centre of the University of Amsterdam

Meibergdreef 9

1100 DD Amsterdam

The Netherlands

Fax: 31206919338

E-mail: P.Dahlem@amc.uva.nl

Keywords: Acute respiratory distress syndrome child

epidemiology

mechanical ventilation

Received: January 112003

Accepted after revision: August 192003
In 1994, an American-European Consensus Conference (AECC) defined the criteria for what has been known as acute respiratory distress syndrome (ARDS) [1] and was first described by AshBAUGH et al. in 1968 [2]. This consensus was necessary because in the past heterogeneous criteria for ARDS were used to include patients in clinical studies, thus precluding comparative studies and definite conclusions. The intention of the AECC was to find a uniform definition to provide more homogeneity and comparability for future research in this field. As a result, acute lung injury (ALI) was introduced as a new term for this disease. This partly replaced the old term ARDS because ALI was considered to more accurately reflect latest insights on the pathophysiological process of this disease. Since then, the term ARDS has been reserved for the most severe form of ALI.

Pathophysiologically, ALI is considered to be an acute inflammatory reaction of the lung with damage to the epithelial-endothelial barrier, causing high permeability pulmonary oedema. Lung compliance is decreased whereas the ventilation/perfusion mismatch increases, resulting in failure of gas exchange. Different intrapulmonary aetiologies, such as pneumonia and aspiration (direct lung injury) and extrapulmonary aetiologies, such as septicaemia and multitrauma (indirect lung injury), may trigger this process.

Since the AECC, studies in adult patients have provided data on the incidence and outcomes of ALI/ARDS [3-5]. No studies have specifically investigated the incidence and outcomes of ALI in children. Only one study, investigating the ARDS subgroup, and another study using a broader definition of acute hypoxic respiratory failure (AHRF), have so far published data on respiratory failure in children. The former was performed on a paediatric intensive care unit (PICU) in Kuala Lumpur, Malaysia [6], and the second one on a PICU in London, UK [7]. Furthermore, the European ARDS network collects epidemiological data on paediatric ARDS from multiple European centres, which are accessible on the internet [8]. Due to the lack of specific data on paediatric ALI, the authors aimed to determine the incidence and short-term outcome of ALI in mechanically ventilated children.

\section{Methods}

After approval from the local Ethics Committee, all admissions from January 11998 to January 12000 were analysed. The PICU is a 12-bed, multidisciplinary, tertiary referral centre and subdivision of the Emma Children's Hospital, Academic Medical Centre of the University of Amsterdam. Children with all types of diseases are admitted based on the following criteria: impending or manifest organ failure of at least one vital organ (respiration, circulation or neurology), patients with a high risk of organ dysfunction and failure due to general paediatric surgery (including multiply injured or neonatal surgical patients) or postorthopaedic surgery, acute peritoneal dialysis or haemodialysis, postcardiac surgery, postneurosurgery and patients whose organ function needs to be closely monitored independent of the underlying disease. The age group of the admitted children ranges from newborns with a birth weight of $>2 \mathrm{~kg}$ 


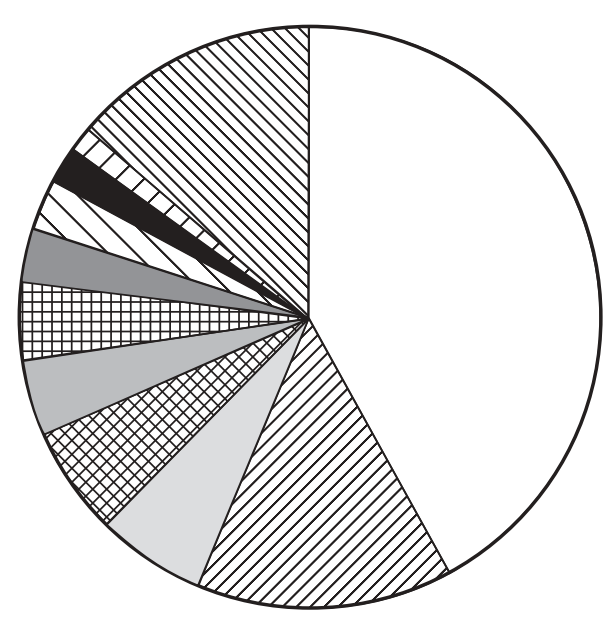

Fig. 1.-Profile of the paediatric intensive care unit population ( $\mathrm{n}=1100)$ admitted during the study period. $\square$ : general paediatrics $(42 \%)$; $\mathbb{Z}$ : paediatric neonatal surgery $(14 \%)$; : neurosurgery $(6 \%)$; ․ cardiology $(6 \%)$; : oncology $(5 \%)$; 眏: cardiac surgery $(4 \%)$; neurology $(3 \%) ; \nabla$ : nephrology $(3 \%) ; \mathbf{\square}$ : otolaryngology $(2 \%) ; \nabla:$ orthopaedics (1\%); $\mathbb{\mathbb { Q }}$ : others (14\%).

(independent of postconceptional age) to adolescents with a maximum age of 18 yrs. Figure 1 presents an overview of the study population (1,100 children) according to the discipline involved.

For the purpose of the study, only mechanically ventilated children who survived the first $24 \mathrm{~h}$ of admission were included. Death and disturbances in gas exchange in the first $24 \mathrm{~h}$ are frequently caused by situations not related to ALI, such as failed resuscitation in septic shock or cerebral herniation (e.g. neurotrauma, cerebral tumours). Furthermore, during this period children are often haemodynamically instable and prone to multiple interventions, such as fluid resuscitation or intubation with aggressive sedation and relaxation. These interventions can aggravate hypoxaemia not related to the pathophysiology of ALI and can therefore lead to a false-positive high incidence of ALI. Post hoc, children who died during the first $24 \mathrm{~h}$ were analysed to determine if they would have fulfilled the AECC criteria in order to assess the degree of underestimation of incidence and mortality of ALI due to this 24-h criterion.

The ventilation strategy of the unit is to ventilate children with ALI in the pressure-controlled mode, with positive endexpiratory pressure (PEEP) titrated based on oxygenation and positive inspiratory pressure (PIP), whenever possible limited to a maximum of $30 \mathrm{cmH}_{2} \mathrm{O}$ with permissive hypercapnia if required. When there is evidence of lung derecruitment, recruitment manoeuvres (e.g. prone positioning, changes in ventilator settings) are performed to improve oxygenation.

All ventilated patients were analysed on the second day of admission, whether or not they fulfilled the AECC criteria for ALI: 1) acute onset; 2) arterial oxygen tension to fraction of inspired oxygen ratio $\left(\mathrm{Pa}, \mathrm{O}_{2} / \mathrm{FI}, \mathrm{O}_{2}\right) \leqslant 40 \mathrm{kPa}$ for $\mathrm{ALI}$ and $\mathrm{Pa}_{2} \mathrm{O}_{2} / \mathrm{FI}, \mathrm{O}_{2} \leqslant 26.7 \mathrm{kPa}$ for $\left.\mathrm{ARDS} ; 3\right)$ no remaining clinical signs of atrial hypertension; and 4) bilateral infiltrates on chest radiographs. For the purpose of the study, criteria 2 and 3 were further elaborated. Firstly, only patients whose $\mathrm{Pa}, \mathrm{O}_{2} /$ $F \mathrm{I}, \mathrm{O}_{2}$ was $<40 \mathrm{kPa}$ in two consecutive measurements with a minimum interval of $8 \mathrm{~h}$ were included. Average $\mathrm{Pa}, \mathrm{O}_{2} / F \mathrm{I}, \mathrm{O}_{2}$ was then calculated for each day from inclusion to extubation or death, based on multiple daily blood-gas analysis (at least every $8 \mathrm{~h}$ ) and the recorded ventilator settings. Secondly, it was diagnosed whether or not left atrial hypertension was clinically present, as suggested by the AECC [1]. As the gold standard of measuring left atrial hypertension with a pulmonary artery catheter is not routinely used in children, cardiac echography was performed when there was doubt about the left ventricular function or when the patients were receiving vasopressor agents. This was performed with the aim to exclude patients with a high risk of cardiogenic pulmonary oedema based on the following criteria: decreased shortening fraction of $<30 \%$, mitral regurgitation and/or enlarged left atrium. The method and the criteria have been described in detail elsewhere $[9,10]$. A paediatric radiologist and a paediatric intensivist, both blinded for clinical information, analysed the chest radiographs for the presence of bilateral infiltrates consistent with lung oedema and interobserver variation was determined.

All patient data (e.g. ventilation methods and requirements, biochemical and physiological variables) were collected prospectively and documented on the charts. The unit policy is to evaluate the diagnosis of ALI in all ventilated children when the $P \mathrm{a}, \mathrm{O}_{2} / F \mathrm{I}, \mathrm{O}_{2}$ falls to $<40 \mathrm{kPa}$. This meant that chest radiographs were performed, if these were not made within the previous $4 \mathrm{~h}$. Furthermore, the hourly documented ventilator settings were linked simultaneously with the blood-gas analysis. As part of the routine, the attending physician also evaluated the haemodynamic condition of the patient and indicated echocardiography, if needed. All these data were documented in the patients' charts and were accessible for analysis. Additionally, a differentiation was made between direct (e.g. pneumonia, aspiration) and indirect (e.g. sepsis, multitrauma, postsurgical) lung injury. The outcome parameter was PICU mortality. The cause of death was recorded as failed resuscitation, untreatable circulatory, respiratory or neurological failure (brain death or severe brain damage) with or without withdrawal of therapy due to a poor prognosis.

For the estimation of severity of disease and for comparison between survivors and nonsurvivors the paediatric index of mortality (PIM) [11], the paediatric risk of mortality score (PRISM) II [12] and a maximum score for multiorgan system failure (MOSF) during hospitalisation were assessed [13]. In addition to the well-developed indices for mortality prediction at admission (i.e. PIM) or $24 \mathrm{~h}$ after admission (i.e. PRISM II), respiratory parameters (e.g. $P \mathrm{a}, \mathrm{O}_{2} / F \mathrm{I}, \mathrm{O}_{2}$ ) shortly after diagnosis of ALI were statistically analysed to determine whether they independently correlated with mortality.

\section{Statistical analysis}

Cohen's kappa was calculated to determine interobserver variation between the paediatric intensivist and radiologist. Collected data on survivors and nonsurvivors were compared. One-sided t-tests (unpaired), Chi-squared tests, Fischer's exact tests, or Mann-Whitney U-tests were used based on the measurement scale and the exploration of distributional characteristics. Significant parameters from the univariate analyses and predictors for mortality (PIM, PRISM II) were selected for multivariate binary logistic regression analysis against survival.

\section{Results}

\section{Incidence}

In the 2-yr study period, 446 of the admitted children were mechanically ventilated. The clinical or radiographical status of three patients were not retrievable, and thus 443 were eligible for analysis. The interobserver agreement between the 
paediatric intensivist and radiologist with respect to judgement of the chest radiographs was excellent (Cohen's kappa of 0.772). All four AECC criteria for ALI were fulfilled by 44 children $(9.9 \%)$. Of these 44 children, 41 were included on admission day 2, and three on day 3; all of them were mechanically ventilated on the first day of admission. In a subgroup analysis, $79.5 \%$ of the ALI patients (35 of 44) were diagnosed as suffering from ARDS. Of these, 54.5\% (24 of 44) immediately fulfilled the ARDS criteria at inclusion and $25 \%$ (11 of 44) developed ARDS later on in the course of their disease. The incidence of ARDS among all mechanically ventilated patients was therefore $7.9 \%$. This subgroup analysis is shown in figure 2 and the patients' characteristics are presented in table 1.

Post hoc, all PICU deaths (36 of 70, 51.4\%) during the first $24 \mathrm{~h}$ were analysed to establish whether they would have been diagnosed as ALI and may have been missed for this study purpose. None of them fulfilled the AECC criteria for ALI, mostly due to cardiac failure.

Of the underlying diseases, meningococcal septic shock (indirect lung injury) was the most frequent (34.1\%) followed by aetiologies with direct lung injury, respiratory syncytial virus infection $(15.9 \%)$ and primary pneumonia $(11.4 \%)$. Four patients $(9.1 \%)$, including those with oncological diseases, were immunocompromised. Twenty-nine children had accompanying MOSF $(65.9 \%)$.

Eight children developed a pneumothorax as a complication; all had ARDS and four of them died. Nine children (all had ARDS) needed one or more of the following specialised intensive care treatments due to intractable respiratory failure: inhaled nitric oxide (nine cases, four died), high frequency oscillatory ventilation (two cases, one died) and extracorporeal membrane oxygenation (one case).

\section{Mortality}

Twelve of the 44 patients died $(27.3 \%)$ and of these 11 had ARDS. The mortality rates in the different subgroups are shown in figure 3 . All except three patients died during the first 8 days after the onset of ALI. In seven patients, the cause of death was severe irreversible cerebral damage, which was caused by the initial insult (i.e. shock, near drowning, smoke inhalation, strangulation). Of the remaining five children, two died due to intractable circulatory failure (septic shock), two

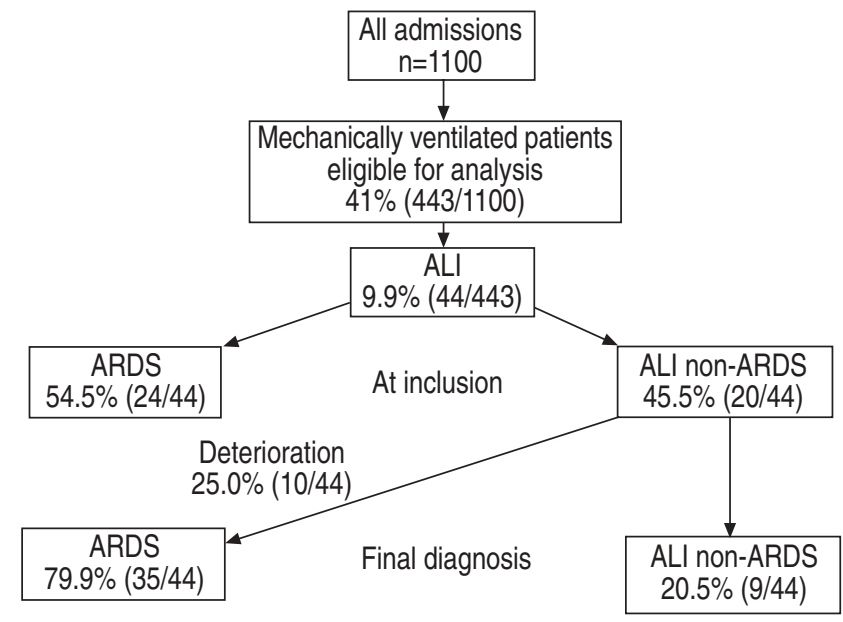

Fig. 2.-Incidences of acute lung injury (ALI) in the subgroups: acute respiratory distress syndrome (ARDS) and ALI non-ARDS.

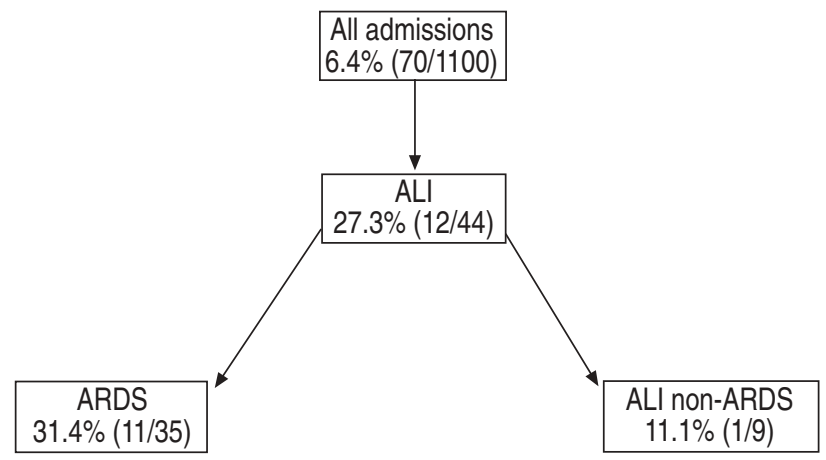

Fig. 3.-Mortality rates of acute lung injury (ALI) and in the subgroups: acute respiratory distress syndrome (ARDS) and ALI non-ARDS.

(4.5\% of ALI) due to intractable respiratory failure (pulmonary haemorrhage, both had a congenital immune disorder) and one died due to veno-occlusive disease. Of the four patients, who were immunocompromised, two died.

Table 1.-Patient characteristics

\begin{tabular}{|c|c|c|c|c|}
\hline & All & Survivors & Nonsurvivors & Test value \\
\hline Subjects n & 44 & 31 & 13 & \\
\hline Age months & $27.5(5.0-58.3)$ & $26.0(3.5-55.8)$ & $36.0(9.0-134.8)$ & $Z=-1.19$ \\
\hline Weight kg & $13.5(6.3-20.0)$ & $12.5(6.3-20.0)$ & $17.5(7.5-43.0)$ & $\mathrm{Z}=-1.10$ \\
\hline Male & $24(54.5)$ & $17(53.1)$ & $7(58.3)$ & $\mathrm{Chi}^{2}=0.10$ \\
\hline PIM & $-2.393 \pm 1.189$ & $-2.519 \pm 1.111$ & $-2.056 \pm 1.373$ & $\mathrm{t}=-1.154$ \\
\hline PRISM & $16.7 \pm 9.6$ & $14.6 \pm 8.7$ & $22.3 \pm 10.1$ & $\mathrm{t}=-2.50^{*}$ \\
\hline Multiorgan system failure & $29(65.9)$ & $17(53.1)$ & $12(100.0)$ & $\mathrm{Chi}^{2}=8.53^{*}$ \\
\hline Direct lung injury & $21(47.7)$ & $15(46.9)$ & $6(50.0)$ & $\mathrm{Chi}^{2}=0.04$ \\
\hline Disease, meningococcus infection or septic shock & $15(34.1)$ & $13(40.6)$ & $2(16.7)$ & $\mathrm{Chi}^{2}=2.23$ \\
\hline ARDS at inclusion & $24(54.5)$ & $15(46.9)$ & $9(75.0)$ & $\mathrm{Chi}^{2}=2.78^{\circ}$ \\
\hline $\mathrm{Pa}, \mathrm{O}_{2} / F \mathrm{I}, \mathrm{O}_{2}$ day 1 after inclusion $\mathrm{kPa}$ & $28.2 \pm 13.8$ & $30.5 \pm 11.9$ & $22.1 \pm 17.2$ & $\mathrm{t}=1.84^{*}$ \\
\hline $\mathrm{Pa}, \mathrm{O}_{2} / F \mathrm{I}, \mathrm{O}_{2}$ day 2 after inclusion $\mathrm{kPa}$ & $27.8 \pm 13.6$ & $28.4 \pm 10.9$ & $26.4 \pm 19.7$ & $\mathrm{t}=0.43$ \\
\hline PIPmax $\mathrm{cmH}_{2} \mathrm{O}$ & $33.1 \pm 7.2$ & $31.7 \pm 6.9$ & $37.0 \pm 6.6$ & $\mathrm{t}=-2.30 *$ \\
\hline PEEPmax $\mathrm{cmH}_{2} \mathrm{O}$ & $9.5 \pm 4.1$ & $8.8 \pm 3.9$ & $11.4 \pm 4.2$ & $\mathrm{t}=-1.96^{*}$ \\
\hline Ventilator days & $6.5(5.3-13.0)$ & $7.0(6.0-13.0)$ & $4.0(2.0-8.0)$ & $\mathrm{Z}=-2.13^{\#}$ \\
\hline
\end{tabular}

Data are presented as median (interquartile range), $\mathrm{n}(\%)$ or mean \pm SD unless otherwise stated. PIM: paediatric index of mortality; PRISM: paediatric risk of mortality; ARDS: acute respiratory distress syndrome; $P \mathrm{a}, \mathrm{O}_{2} / F \mathrm{I}, \mathrm{O}_{2}$ : average arterial oxygen tension to fraction of inspired oxygen ratio; PIPmax: positive inspiratory pressure; PEEPmax: positive end-expiratory pressure. The Z-value is derived from Mann-Whitney U-tests and the t-tests were unpaired. *: p $<0.05$ one-sided; ${ }^{\#}$ : $\mathrm{p}<0.05$ two-sided; ${ }^{\uparrow}$ : $\mathrm{p}=0.05-0.10$ one-sided. 
Table 1 presents the results of the univariate analysis comparing nonsurvivors with survivors. Significant differences for nonsurvivors and survivors were found for the PRISM, MOSF, $P$ a $, \mathrm{O}_{2} / F \mathrm{I}, \mathrm{O}_{2}$ on day 1 after inclusion, maximal PIP and maximal PEEP. Due to the small number of patients $(n=44)$ and to prevent multicollinearity, only three parameters were analysed from this set in the "best" multivariate binary logistic regression model: PIM, PRISM and $\mathrm{Pa}, \mathrm{O}_{2} / F I, \mathrm{O}_{2}$ on day 1 after inclusion. The $\mathrm{Pa}, \mathrm{O}_{2} / F \mathrm{I}, \mathrm{O}_{2}$ on day 1 after inclusion and PRISM II were associated with mortality with an area under the receiver operating characteristic curve (AUC) of 0.728 and 0.732 , respectively (table 2). Combining both, the AUC improved to 0.815 compared with each parameter alone.

\section{Discussion}

To the best of the authors' knowledge, this is the first report specifically detailing the incidence, patients' characteristics and outcome of paediatric ALI in a Western European PICU. The basic findings are: 1) an incidence of $9.9 \%$ of ALI of the ventilated children with $80 \%$ of them developing ARDS; 2) a higher mortality in the ARDS subgroup compared with the ALI non-ARDS subgroup; and 3) $25 \%$ of children with ALI at inclusion deteriorated later to ARDS. As there is a lack of precise data in the literature, it seems appropriate in some cases to make comparisons with surveys that have investigated comparable groups of children with similar pathology (AHRF/ARDS), whose inclusion criteria were also based on the AECC. In case such data were unavailable, comparisons with adult studies have been made.

Only the incidence of ARDS, as the largest subgroup of ALI patients, has been studied so far. In an Asian population an incidence of ARDS of $4.1 \%$ was found [6], which was higher than the $3.2 \%$ (35 of 1,100) in the present PICU population (fig. 2). This difference may be explained by higher admission thresholds, higher severity scores for nonsurvivors (PRISM 30.4 versus 22.3) and higher incidences of infections (e.g. sepsis $61.5 \%$ versus $16.7 \%$ ) in the Asian study.

A total of $79.5 \%$ of the present ALI patients developed ARDS. At inclusion, however, only 54.5\% had ARDS and $25 \%$ developed ARDS later in the course of their disease. This observation supports one of the intentions of the AECC, whereby ALI was introduced to allow early diagnosis of the disease for scientific and clinical purposes [1,3,4]. The $25 \%$ of patients who deteriorated to ARDS could be an important target population for novel therapies (e.g. protective ventilation strategies, drug therapy) in order to prevent the development of ARDS and to improve outcome [14, 15]. Furthermore, the causes of deterioration should be studied in the context of ventilator-induced lung injury (VILI) and MOSF $[15,16]$. The high percentage $(79.5 \%)$ of children with ALI, who finally developed ARDS was similar to the data from two large adult studies $(76-80 \%)[3,4]$.

ALI represents severe respiratory failure and is frequently part of MOSF. The incidence of MOSF in the ALI population $(65.9 \%)$ was almost twice as high as the $33.9 \%$ in a study that used a more general definition of paediatric AHRF
[7]. This difference could be explained by the fact that their definition of AHRF also included children without infiltrates on the chest radiograph (criterion 4), resulting in the inclusion of less severely ill children.

As mentioned above, inclusion was started $24 \mathrm{~h}$ after admission in order to avoid an overestimation of the incidence of ALI. Furthermore, death shortly after admission may be caused by the initial insult rather than by deteriorating respiratory failure. In order to elucidate a possible bias selection due to this criterion, a post hoc analysis of all PICU deaths (36 of 70, 51.4\%) was performed during the first $24 \mathrm{~h}$ to establish whether or not they would have been diagnosed as ALI and may have been missed for the analysis. However, none of them fulfilled the AECC criteria for ALI before death.

Neither specific mortality data for paediatric ALI nor standardised mortality ratios (SMR; i.e. observed deaths divided by expected deaths) have yet been published. With respect to the subgroup ARDS, however, two European investigations reported crude mortality rates of $32 \%$ and $36.5 \%[7,8]$, which are similar to the $31.4 \%$ found in the present study, and in an Asian population a mortality rate double that found in Europe was reported (62\%) [6]. An appropriate interpretation of the differences in mortality rates between these studies can only be made by the comparison of the SMR for each unit. However, published data do not allow the calculation of SMRs. Therefore, it can only be hypothesised that the differences in mortality rates may be caused by differences in patient populations between these studies. For example, when compared with this study, GoH et al. [6] reported, within the same group of patients (all were mechanically ventilated and fulfilled the AECC criteria for ARDS), more sepsis patients (61.5\% versus $16.7 \%)$ and higher PRISM scores (30.4 versus 22.3) among the nonsurvivors.

For the western part of the world, mortality in adult and paediatric ARDS patients has been declining in the last decade to $30-40 \%$, which has been attributed to an overall improvement of intensive care treatment and the changes in ventilation strategies [17-21]. When the subgroups within the present ALI population were compared, it was observed that of the 12 children who died, only one of nine was in the ALI non-ARDS group, whereas 11 of 35 were in the ARDS group (fig. 3). This is in contrast to the adult literature, where mortality did not differ between the ALI non-ARDS and the ARDS subgroups [5].

In the present study, the leading cause of death was cerebral damage (seven of 12) suffered as a result of the initial insult. Only two patients died due to pulmonary complications and both had pre-existing complex congenital immune disorders. This suggests that the initial trigger negatively influences further organ dysfunction and final outcome. One paediatric study on AHRF and multiple adult studies showed that underlying diseases, such as cerebral dysfunction and immune disorders, have a greater impact on outcome than respiratory failure alone [7, 18, 22-28]. These findings suggest that ALI may be more a symptom than a limiting disease. However, this seems to contradict the strong evidence that ongoing pulmonary inflammation during ALI, and secondarily caused by VILI, triggers a de novo systemic inflammatory response,

Table 2. - Data on multivariate regression analysis

\begin{tabular}{lcccr}
\hline Factor & $\beta$ & SE & Exp $(\beta)$ & $95 \%$ CI \\
\hline PRISM & 0.097 & 0.04 & 1.101 & $1.019-1.190$ \\
$P \mathrm{a}, \mathrm{O}_{2} / F \mathrm{I}, \mathrm{O}_{2}$ day 1 after inclusion & -0.099 & 0.03 & 0.906 & $0.854-0.961$ \\
\hline
\end{tabular}

CI: confidence interval; PRISM: paediatric risk of mortality; $\mathrm{Pa}, \mathrm{O}_{2} / F \mathrm{I}, \mathrm{O}_{2}$ : arterial oxygen tension to fraction of inspired oxygen ratio (average score on day 1 after inclusion). McFadden's- $\mathrm{R}^{2}=33.4 \%$. Overall per cent correctly predicted 86.4 . Area under the curve $\left(\right.$ combination of $P$ a, $\mathrm{O}_{2} / F \mathrm{I}, \mathrm{O}_{2}$ on day 1 after inclusion and PRISM) 0.815 (95\% CI 0.642-0.989). 
leading to multiple organ failure and an increase in mortality $[15,16]$. Future studies in children should therefore define the clinical situations in which ALI is just a symptom and resolves together with the underlying disease, or a contributing factor for multiorgan failure and negative outcome.

In this context, it would be desirable to have parameters for decision-making at the bedside and for selection of patients for new therapies and clinical trials. Respiratory parameters (e.g. oxygenation indices) may be suitable because they represent pulmonary function and indicate deterioration of ALI, before a systemic response is initiated and outcome negatively affected.

One of the most frequently studied indices is the $\mathrm{Pa}, \mathrm{O}_{2} /$ $F \mathrm{I}, \mathrm{O}_{2}$, although contradictory results concerning its ability to predict outcome have been reported in adult AHRF/ARDS [2, 27-35]. Some authors have argued that the ratio may not be predictive at inclusion, but after 24 or $48 \mathrm{~h}[30,31]$. Two paediatric studies on children with generalised AHRF also failed to clearly define respiratory parameters for outcome prediction $[6,7]$.

Due to the small number of children with ALI $(n=44)$ in the present study, the number of respiratory parameters for multilogistic regression analysis had to be limited. Therefore, only those parameters that can be early and easily performed at the bedside were focused upon $\left(\mathrm{Pa}, \mathrm{O}_{2} / F \mathrm{I}, \mathrm{O}_{2}\right.$ on day 1 and 2 after inclusion). The $\mathrm{Pa}, \mathrm{O}_{2} / \mathrm{FI}, \mathrm{O}_{2}$ was compared with two standardised scoring systems for mortality prediction in children (i.e. PIM and PRISM). The PIM is performed at admission and accounts for the pre-existing condition of the patient, whereas the PRISM is performed $24 \mathrm{~h}$ after admission and also reflects the progression of disease during the first hours. The analysis revealed that the AUC of the $P$ a, $\mathrm{O}_{2} /$ $F \mathrm{I}, \mathrm{O}_{2}$ on day 1 after inclusion was similar to the AUC of the PRISM, and that the combination of both resulted in an improvement of the AUC (0.815). Interestingly, the PIM did not discriminate between survivors and nonsurvivors of ALI. Two possible explanations for the findings were considered. Firstly, the PIM may not be reliable for specific diagnostic subgroups such as ALI, because it was developed for a PICU population as a whole [11]. This explanation is supported by a recent report, where the PIM of children with respiratory failure showed a smaller AUC than the overall PICU population [36]. Secondly, another reason may be that the PIM of the ALI nonsurvivors was very low $-2.056 \pm 1.373$. This may be explained by the fact that they survived the first $24 \mathrm{~h}$ (inclusion criterion for the diagnose of ALI) and therefore may belong to a group of patients with a lower PIM score compared with other PICU patients, who died during the first $24 \mathrm{~h}$ (i.e. early nonsurvivors). Indeed, PICU patients who died during the first $24 \mathrm{~h}$ had a significantly higher PIM score of $0.622 \pm 2.652$ compared with the nonsurvivors with ALI $(p<0.004)$. In other words, the late nonsurvivors of the ALI patients had been less severely ill at admission than the early nonsurvivors of the whole PICU population. Obviously the most severely ill children on a PICU die on the first day and for them PIM is a reliable predictor, whereas for nonsurvivors with ALI, who die later, the PIM loses reliability.

In summary, due to the small number of patients with ALI and the limited number of parameters used for regression analysis, these results only suggest a possible association between a respiratory parameter $\left(\mathrm{Pa}, \mathrm{O}_{2} / F \mathrm{I}, \mathrm{O}_{2}\right)$ and outcome. Therefore, future studies should confirm this association, considering the vicious circle of lung injury: localised pulmonary inflammation due to an initial trigger (e.g. pneumonia), secondary lung damage due to mechanical ventilation, and finally the development of a systemic inflammatory response negatively influencing outcome.

To conclude, acute lung injury and acute respiratory distress syndrome are uncommon diseases in a paediatric intensive care unit but have a high mortality. Approximately $80 \%$ of children with acute lung injury develop acute respiratory distress syndrome. The mortality in the acute respiratory distress syndrome subgroup is higher than in the acute lung injury nonacute respiratory distress syndrome subgroup and is determined by the underlying diseases. Future investigations should confirm what factors indicate the deterioration from acute lung injury to acute respiratory distress syndrome and predict outcome. Children who are at risk can then be identified and may benefit from novel therapeutic approaches.

Acknowledgements. The authors would like to thank A. Smets (paediatric radiologist) for interpreting the chest radiographs and $M$. de Neef (research nurse) for helping with data collection.

\section{References}

1. Bernard GR, Artigas A, Brigham KL, et al. The AmericanEuropean Consensus Conference on ARDS. Definitions, mechanisms, relevant outcomes, and clinical trial coordination. Am J Respir Crit Care Med 1994; 149: 818-824.

2. Ashbaugh DG, Bigelow DB, Petty TL, Levine BE. Acute respiratory distress in adults. Lancet 1967; 2: 319-323.

3. Zilberberg MD, Epstein SK. Acute lung injury in the medical ICU, comorbid conditions, age, etiology and hospital outcome. Am J Respir Crit Care Med 1998; 157: 1159-1164.

4. Roupie E, Lepage E, Wysocki M, et al. Prevalence, etiologies and outcome of the acute respiratory distress syndrome among hypoxemic ventilated patients. Intensive Care Med 1999; 25: 920-929.

5. Luhr OR, Antonsen K, Karlsson M, et al. Incidence and mortality after acute respiratory failure and acute respiratory distress syndrome in Sweden, Denmark, and Iceland. The ARF Study Group. Am J Respir Crit Care Med 1999; 159: 1849-1861.

6. Goh AYT, Chan PWK, Lum LCS, Roziah M. Incidence of acute respiratory distress syndrome: a comparison of two definitions. Arch Dis Child 1998; 79: 256-259.

7. Peters MJ, Tasker RC, Kiff KM, Yates R, Hatch D. Acute hypoxemic respiratory failure in children: case mix and the utility of respiratory severity indices. Intensive Care Med 1998; 24: 699-705.

8. Bindl L, Betancort M, Demirakca S, et al. ARDS database in children: data of 91 patients collected by a collaborative internet database. http://www.meb.uni-bonn. Date last accessed: February 9, 2001.

9. Hagmolen W, Wiegman A, van den Hoek GJ, Vreede WB, Derkx HHF. Life-threatening heart failure in meningococcal septic shock in children: non-invasive measurement of cardiac parameters is of important prognostic value. Eur J Pediatr 2000; 159: 277-282.

10. Kimball TR, Meyer RA. Echocardiography. In: Allen HD, ed. Moss and Adam's Heart Disease in Infants, Children, and Adolescents. 6rd Edn. Philadelphia, Lippincott Williams and Wilkins, 2001; pp. 204-233.

11. Shann F, Pearson G, Slater A, Wilkinson K. Paediatric index of mortality (PIM): a mortality prediction model for children in intensive care. Intensive Care Med 1997; 23: 201-207.

12. Pollack MM, Ruttimann UE, Getson PR. Pediatric risk of mortality (PRISM) score. Crit Care Med 1988; 16: 11101116.

13. Wilkinson JD, Pollack MM, Ruttimann UE, Glass NL, Yeh TS. Outcome of pediatric patients with multiple organ system failure. Crit Care Med 1986; 14: 271-274.

14. Meduri GU, Tolley EA, Chrousos GP, Stentz F. Prolonged methylprednisolone treatment suppresses systemic 
inflammation in patients with unresolving acute respiratory distress syndrome: evidence for inadequate endogenous glucocorticoid secretion and inflammation-induced immune cell resistance to glucocorticoids. Am J Respir Crit Care Med. 2002; 165: 983-991.

15. The Acute Respiratory Distress Syndrome Network. Ventilation with lower tidal volumes as compared with traditional tidal volumes for acute lung injury and the acute respiratory distress syndrome. $N$ Engl J Med 2000; 342: 1301-1308.

16. Slutsky AS, Tremblay LN. Multiple system organ failure. Is mechanical ventilation a contributing factor? Am J Respir Crit Care Med 1998; 157: 1721-1725.

17. Wyncoll DLA, Evans TW. Acute respiratory distress syndrome. Lancet 1999; 354: 497-501.

18. Abel SJC, Finney SJ, Brett SJ, Keogh BF, Morgan CJ, Evans TW. Reduced mortality in association with the acute respiratory distress syndrome (ARDS). Thorax 1998; 53: 292-294.

19. Jardin F, Fellahi JL, Beauchet A, Vieillard-Baron A, Loubieres Y, Page B. Improved prognosis of acute respiratory distress syndrome 15 years on. Intensive Care Med 1999; 25: $936-941$.

20. Milberg JA, Davis DR, Steinberg KP, Hudson LD. Improved survival of patients with acute respiratory distress syndrome (ARDS): 1983-1993. JAMA 1995; 273: 306-309.

21. Lewandowski K. Readers' comments in focus. Intensive Care Med 2000; 26: 845-847.

22. Squara P, Dhainaut JFA, Artigas A, Carlet J, and the European Collaborative ARDS Working Group. Hemodynamic profile in severe ARDS: results of the European Collaborative ARDS study. Intensive Care Med 1998; 24: 1018-1028.

23. Tantalean JA, Leon RJ, Santos AA, Sanchez E. Multiple organ dysfunction syndrome in children. Pediatr Crit Care Med 2003; 4: 181-185.

24. Cengiz P, Zimmerman JJ. Prelude to pediatric multiple organ dysfunction syndrome: the golden hours concept revisited. Pediatr Crit Care Med 2003; 4: 263-264.
25. Lyrene RK, Truog WE. Adult respiratory distress syndrome in a pediatric intensive care unit: predisposing conditions, clinical course and outcome. Pediatrics 1981; 67: 790-795.

26. Ware LB, Matthay MA. The acute respiratory distress syndrome. N Engl J Med 2000; 342: 1334-1349.

27. Jiminez P, Torres A, Roca J, Cobos A, Rodriguez-Roison R. Arterial oxygenation does not predict the outcome of patients with acute respiratory failure needing mechanical ventilation. Eur Respir J 1994; 7: 730-735.

28. Zimmerman JE, Knaus WA, Wagner DP, Sun X, Hakim $\mathrm{RB}$, Nystrom PO. A comparison of risks and outcomes for patients with organ system failure: 1982-1990. Crit Care Med 1996; 24: 1633-1641.

29. Doyle RL, Szaflarski N, Modin GW, Wiener-Kronish JR, Matthay MA. Identification of patients with acute lung injury. Predictors of mortality. Am J Respir Crit Care Med 1995; 152: 1818-1824.

30. Villar J, Perez-Mendez L, Kacmarek RM. Current definitions of acute lung injury and the acute respiratory distress syndrome do not reflect their true severity and outcome. Intensive Care Med 1999; 25: 930-935.

31. Davis SL, Furman DP, Costarino AT. Adult respiratory distress syndrome in children: associated disease, clinical course and predictors of death. J Pediatr 1993; 123: 35-45.

32. Ferring M, Vincent JL. Is outcome of ARDS related to severity of respiratory failure? Eur Respir J 1997; 10: 12971230.

33. Nolan S, Burgess K, Hopper L, Braude S. Acute respiratory distress syndrome in a community hospital ICU. Intensive Care Med 1997; 23: 530-538.

34. Knaus WA. The ongoing mystery of ARDS. Intensive Care Med 1996; 22: 517-518.

35. Valta P, Uusaro A, Nunes S, Ruokonen E, Takala J. Acute respiratory distress syndrome: frequency, clinical course and costs of care. Crit Care Med 1999; 27: 2367-2374.

36. Slater A, Shann F, Pearson G. PIM2: a revised version of the Paediatric Index of Mortality. Intensive Care Med 2003; 29: 278-285. 